\title{
COVID-19 e tabagismo: uma relação de risco
}

\author{
COVID-19 and smoking: a high-risk association
}

COVID-19 y tabaquismo: una relación de riesgo

Andre Luiz Oliveira da Silva 1,2
Josino Costa Moreira 2
Stella Regina Martins ${ }^{3}$

doi: 10.1590/0102-311X00072020
O SARS-CoV-2 é um novo tipo de coronavírus, causador da COVID-19, que emergiu na China no final de 2019 e alcançou status de pandemia rapidamente. Esse vírus tem tropismo pelo sistema respiratório, causando desde uma síndrome respiratória aguda, que na grande maioria dos casos se manifesta de forma leve, evoluindo em alguns casos para um tipo gravíssimo e célere de pneumonia com insuficiência respiratória importante, progredindo para óbito ${ }^{1}$.

Entre os fatores de risco para desfechos mais graves como a necessidade de internação em unidade de terapia intensiva (UTI) e/ou uso de ventilação mecânica e morte para a COVID-19 estão: idade de 65 anos ou mais; pessoas internadas em instituições de longa permanência; pacientes com doença pulmonar obstrutiva crônica, asma (moderada a grave) e dependentes de oxigênio; pessoas com problemas cardíacos graves ou descompensados; hipertensos descompensados; diabéticos; portadores de doenças cromossômicas ou estado de fragilidade imunológica; indivíduos com insuficiência renal crônica avançada; gestantes de alto risco; pessoas de qualquer idade com obesidade grave (IMC > 40); e condições médicas, como doenças hepáticas 2,3. É possível ainda que a COVID-19 seja uma doença endotelial, podendo levar a quadros graves de coagulopatias e tromboses 4 .

Entretanto, é possível que um importante fator de risco para a COVID-19 não esteja sendo incluído nas recomendações globais para o controle da pandemia, o tabagismo.

Fumantes fazem parte do grupo de risco para a contaminação pela COVID-19. É plausível inferir sobre o aumento do risco de contaminação desse grupo na medida em que fumantes levam os produtos de tabaco, quer sejam tradicionais ou dispositivos eletrônicos para fumar (DEFs) à boca, sem a adequada higienização das mãos.

Os narguilés, muito populares entre a população jovem, que geralmente compartilha os bocais, facilitam a transmissão da COVID-19 5. A Organização Mundial da Saúde (OMS) demonstrou preocupação com o potencial para a disseminação da doença por intermédio do uso dos produtos de tabaco ${ }^{5}$. A literatura indica que doenças como influenza, herpes labial e tuberculose são transmitidas por bocais do narguilé 6 . Por suas características que possibilitam seu compartilhamento, os DEFs também devem ser incluídos como produtos que contribuem para a contaminação pelo SARS-CoV-2.

Outro fato relevante é o risco do fumante sofrer queimaduras ao acender o cigarro ou manusear o narguilé pós-higienização das mãos com álcool em gel, haja vista ser este produto altamente inflamável.
${ }_{1}^{1}$ Agência Nacional de Vigilância Sanitária, Rio de Janeiro, Brasil.

2 Escola Nacional de Saúde Pública Sergio Arouca, Fundação Oswaldo Cruz, Rio de Janeiro, Brasil. 3 Instituto do Coração do Hospital das Clínicas da Faculdade de Medicina da Universidade de São Paulo, São Paulo, Brasil.

\section{Correspondência}

A. L. O. Silva

Laboratório de Toxicologia,

Centro de Estudos da Saúde do Trabalhador e Ecologia Humana, Escola Nacional de Saúde Pública Sergio Arouca, Fundação Oswaldo Cruz. Rua Leopoldo Bulhões 1480, Rio de Janeiro, RJ 21041-210,Brasil. andre.sp.ensp@gmail.com 
Em relação aos danos causados pela infecção da COVID-19, fumar aumenta o risco de danos pulmonares. O tabagismo está relacionado à bronquiolite respiratória (geralmente assintomática), com diversos tipos de pneumonias, além da bronquite crônica, enfisema pulmonar, tuberculose e cânceres de pulmão, promovendo o declínio da função pulmonar ${ }^{7}$.

Estudos em animais e em células humanas sugerem que o tabaco aumenta a gravidade de doenças como a infecção por influenza, aumentando a replicação viral pela supressão dos mecanismos antivirais e alterações nos padrões de citocinas em células com papel central na imunidade inata da mucosa 5. Somando-se a isso, o tabagismo também aumenta a expressão da enzima conversora de angiotensina 2 (ECA2), conhecido receptor do SARS-CoV-2 8

O tabagismo tem relação com a disfunção endotelial e a elevação da concentração dos radicais livres, da mesma forma que infecções microbianas como a COVID-19 9. É plausível acreditar que a COVID-19 possa ocasionar danos ao endotélio previamente lesionado do fumante. Entretanto, a cessação do tabagismo melhora significativamente a função endotelial 10 .

Pacientes com COVID-19 apresentaram níveis elevados de proteína C reativa (PCR) e dímero-D 6,7. Esses marcadores de diagnóstico de trombose encontram-se também alterados em fumantes 11,12. Um estudo observou quadro de coagulação intravascular disseminada em $71 \%$ dos óbitos por COVID-19 comparado com 0,4\% dos sobreviventes ${ }^{13}$. Níveis mais altos de dímero-D (acima de $1 \mu \mathrm{g} / \mathrm{L}$ ) na admissão aumentam em 18 vezes a chance de evolução para óbito 14,15 . É desconhecido o mecanismo dessas complicações, mas elas sugerem a relevância da relação do impacto do tabagismo no endotélio e a COVID-19.

Apesar de uma metanálise não ter encontrado relação entre o tabagismo e os desfechos mais graves da COVID-19 14, o artigo mais robusto e com maior população estudada apontou relação entre o fumar e um quadro mais sombrio de progressão da doença 15 . Artigos em preprints sugerem que o tabagismo poderia ser um fator de risco para manifestações graves da COVID-19 13,16. Somando-se a isso, uma metanálise publicada mais recentemente aponta que o tabagismo é um fator de risco para o prognóstico da COVID-19 17.

Fumantes com COVID-19 têm 3,25 vezes mais chances de desenvolver quadros mais graves da doença do que não fumantes 18 .

Apesar da plausibilidade de que essas complicações possam ser explicadas pelos impactos do tabagismo, alguns estudos não demonstraram a relação entre o tabagismo e a progressão da COVID-19.

As possíveis explicações para tais fatos podem ser observadas no artigo de Szklo 19, em que são listadas: a incorreta identificação dos fumantes, vulnerabilidade econômica, maior atenção à exposição ao vírus e a menor frequência em ambientes fechados devido às leis de ambientes livres. Em adição as essas, poderíamos incluir: a definição de fumantes utilizada (p.ex.: considerar usuários de cigarros eletrônicos como não fumantes e quanto tempo desde o último uso de tabaco) e o viés de informação, haja vista a quase ausente comunicação entre profissionais de saúde, familiares e pacientes nos casos mais graves.

No caso dos DEFs, não existe estudo que correlacione a progressão da COVID-19 e o consumo destes. Entretanto, no caso dos cigarros eletrônicos estudos em animais demonstraram alteração na homeostase lipídica dos macrófagos alveolares e células epiteliais e diminuição da imunidade contra agentes virais 20 .

Em humanos, foi relatado que usuários de cigarros eletrônicos apresentaram alterações no perfil de proteínas ligadas à defesa imunológica inata nas secreções das vias aéreas, induzindo alterações semelhantes às observadas nos fumantes tradicionais 21.

Em relação aos produtos de tabaco aquecido, estudos independentes sugerem que estes produtos causariam alterações na homeostase das células pulmonares 22 e danos endoteliais 23 . Tantos esses produtos como os cigarros eletrônicos poderiam também aumentar a expressão do receptor viral ECA2 8.

Assim, as alterações e danos pulmonares causados pelo uso de produtos do tabaco, incluindo os DEFs, poderiam ser considerados fatores de risco para manifestações e evoluções mais graves da COVID-19.

Com a chegada da pandemia, as incertezas futuras e as medidas de isolamento social se associam à preocupação com a doença. Nesse cenário, a população pode ter respostas emocionais que irão interferir de forma negativa na autoeficácia, por se sentir emocionalmente fragilizada diante de uma 
real ameaça, podendo recorrer às estratégias inadequadas, como fumar, na tentativa de reduzir o desequilíbrio emocional 24.

Apesar de não existirem dados sobre tabagismo e sua recaída em relação às epidemias, estudos apontam que fumantes expostos a desastres naturais fumam mais do que fumantes não expostos $25 \mathrm{e}$ afetam também a recaída de ex-fumantes 26.

No isolamento social e a indicação para que as pessoas fiquem em suas casas, os fumantes expõem os não fumantes às emissões destes produtos. $\mathrm{O}$ fumo passivo pode causar danos semelhantes ao tabagismo ativo 27, incluindo aumentar a expressão da ECA2 8. Não se pode descartar a possibilidade da emissão de aerossóis contendo o vírus, em especial nos DEFs considerando sua menor temperatura de operação. Mais pesquisas são necessárias para responder à extensão dos impactos do fumo passivo na progressão e transmissão da COVID-19.

As trocas gasosas, a função pulmonar e a circulação sanguínea, processos diretamente afetados na COVID-19, melhoram em curto espaço de tempo após a cessação do tabagismo 10. Parar de fumar e não se expor à fumaça e vapores do tabaco pode impactar positivamente na redução da relação de risco entre COVID-19 e tabagismo.

\section{Colaboradores}

Todos os autores colaboraram na elaboração e revisão final do texto.

\section{Informações adicionais}

ORCID: Andre Luiz Oliveira da Silva (0000-00034768-959X); Josino Costa Moreira (0000-00027457-2920); Stella Regina Martins (0000-00033321-8519).

\section{Agradecimentos}

Os autores gostariam de agradecer à Agência Nacional de Vigilância Sanitária (Anvisa), à Fundação Oswaldo Cruz (Fiocruz), ao Instituto do Coração do Hospital das Clínicas da Faculdade de Medicina da Universidade de São Paulo (InCor/HC/ FMUSP) e ao Conselho Nacional de Desenvolvimento Científico e Tecnológico (CNPq). Este artigo representa única e exclusivamente as opiniões e os pensamentos dos autores, baseados nas evidências científicas disponíveis no momento. Eles não representam qualquer diretriz e/ou opinião institucional da Anvisa, do InCor/HC/FMUSP, da Fiocruz, do Ministério da Saúde ou do Governo Brasileiro.

\section{Referências}

1. World Health Organization. Coronavirus disease (COVID-19) pandemic. https://www.who. int/emergencies/diseases/novel-coronavirus2019 (acessado em 14/Abr/2020).

2. Wu C, Chen X, Cai Y, Xia J, Zhou X, Xu S, et al. Risk factors associated with acute respiratory distress syndrome and death in patients with coronavirus disease 2019 pneumonia in Wuhan, China. JAMA Intern Med 2020; [Epub ahead of print].

3. Ministério da Saúde. Guia de Vigilância Epidemiológica. Emergência de saúde pública de importância nacional pela doença pelo coronavírus 2019. Vigilância integrada de síndromes respiratórias agudas, doença pelo coronavírus 2019, influenza e outros vírus respiratórios. https://www.saude.gov.br/images/pdf/2020/ April/06/GuiaDeVigiEp-final.pdf (acessado em 14/Abr/2020)

4. Sardu C, Gambardella J, Morelli MB, Wang X, Marfella R, Santulli G. Is COVID-19 an endothelial disease? Clinical and basic evidence. Preprints 2020; 2020040204. https://www.pre prints.org/manuscript/202004.0204/v1.

5. WHO Framework Convention on Tobacco Control. Increased risk of COVID-19 infection amongst smokers and amongst waterpipe users. https://untobaccocontrol.org/kh/water pipes/covid-19/ (acessado em 31/Mar/2020).

6. Instituto Nacional de Câncer José Alencar Gomes da Silva. Narguilé: o que sabemos? Rio de Janeiro: Instituto Nacional de Câncer José Alencar Gomes da Silva; 2019.

7. U.S. Department of Health and Human Services. The health consequences of smoking: 50 years of progress. A report of the Surgeon General. Atlanta: U.S. Department of Health and Human Services; 2014. 
8. Brake SJ, Barnsley K, Lu W, McAlinden KD, Eapen MS, Sohal SS. Smoking upregulates angiotensin-converting enzyme-2 receptor: a potential adhesion site for novel coronavirus SARS-CoV-2 (Covid-19). J Clin Med 2020; 9:E841.

9. Evora PRB, Baldo CF, Celotto AC, Capellini VK. Endothelium dysfunction classification: why is it still an open discussion? Int J Cardiol 2009; 137:175-6.

10. U.S. Department of Health and Human Services. Smoking cessation: a report of the Surgeon General. Atlanta: U.S. Department of Health and Human Services; 2020.

11. Das I. Raised C-reactive protein levels in serum from smokers. Clin Chim Acta 1985; 153:9-13.

12. Lee AJ, Fowkes GR, Lowe GD, Rumley A. Determinants of fibrin D-dimer in the Edinburgh Artery Study. Arterioscler Thromb Vasc Biol 1995; 15:1094-7.

13. Cai G. Bulk and single-cell transcriptomics identify tobacco-use disparity in lung gene expression of ACE2, the receptor of 2019-nCov. Preprints 2020; 2020020051. https://www.pre prints.org/manuscript/202002.0051/v2.

14. Vardavas CI, Nikitara K. COVID-19 and smoking: a systematic review of the evidence. Tob Induc Dis 2020; 18:20.

15. Cai H. Sex difference and smoking predisposition in patients with COVID-19. Lancet Respir Med 2020; 8:e20.

16. Alqahtani JS, Oyelade T, Aldhahir AM, Alghamdi SM, Almehmadi M, Alqahtani AS, et al. Prevalence, severity and mortality associated with COPD and smoking in patients with COVID-19: a rapid systematic review and meta-analysis. medRxiv 2020; 27 mar. https:// www.medrxiv.org/content/10.1101/2020.03.2 $5.20043745 \mathrm{v} 1$.

17. Patanavanich R, Glantz SA. Smoking is associated with COVID-19 progression: a metaanalysis. medRxiv 2020; 16 abr. https://www. medrxiv.org/content/10.1101/2020.04.13.200 $63669 \mathrm{v} 1$.

18. Guan W, Ni Z, Hu Y, Liang W, Ou C, He J, et al. Clinical characteristics of coronavirus disease 2019 in China. N Engl J M 2020; 382:1708-20.
19. Szklo AS. Associação entre fumar e progressão para complicações respiratórias graves em pacientes com Covid-19. Rev Bras Cancerol 2020; 66:e-03974.

20. Madison MC, Landers CT, Gu B-H, Chang C-Y, Tung H-Y, You R, et al. Electronic cigarettes disrupt lung lipid homeostasis and innate immunity independent of nicotine. J Clin Invest 2019; 129:4290-304

21. Reidel B, Radicioni G, Clapp PW, Ford AA, Abdelwahab S, Rebuli ME, et al. E-cigarette use causes a unique innate immune response in the lung, involving increased neutrophilic activation and altered mucin secretion. Am J Respir Crit Care Med 2018; 197:492-501.

22. Sohal SS, Eapen MS, Naidu VGM, Sharma P. IQOS exposure impairs human airway cell homeostasis: direct comparison with traditional cigarette and e-cigarette. ERJ Open Res 2019; 5:00159-2018.

23. Nabavizadeh P, Liu J, Havel CM, Ibrahim S, Derakhshandeh R, Jacob III P, et al. Vascular endothelial function is impaired by aerosol from a single IQOS HeatStick to the same extent as by cigarette smoke. Tob Control 2018; 27 Suppl 1:s13-9.

24. Salvetti MG, Pimenta CAM. Dor crônica e a crença de auto-eficácia. Rev Esc Enferm USP 2007; 41:135-40.

25. Huh J, Timberlake DS. Do smokers of specialty and conventional cigarettes differ in their dependence on nicotine? Addict Behav 2009; 34:204-11.

26. Lanctot JQ, Stockton MB, Mzayek F, Read M, McDevitt-Murphy M, Ward K. Effects of disasters on smoking and relapse: an exploratory study of Hurricane Katrina victims. Am J Health Educ 2008; 39:91-4.

27. U.S. Department of Health and Human Services. The health consequences of involuntary exposure to tobacco smoke: a report of the Surgeon General. Atlanta: U.S. Department of Health and Human Services; 2006.
Recebido em 06/Abr/2020

Versão final reapresentada em 27/Abr/2020

Aprovado em 28/Abr/2020 